\title{
Video Article \\ Clinical Examination Protocol to Detect Atypical and Classical Scrapie in Sheep
}

\author{
Timm Konold ${ }^{1}$, Laura Phelan ${ }^{1}$ \\ ${ }^{1}$ Specialist Scientific Support Department, Animal Health and Veterinary Laboratories Agency Weybridge \\ Correspondence to: Timm Konold at Timm.Konold@ahvla.gsi.gov.uk
}

URL: https://www.jove.com/video/51101

DOI: doi:10.3791/51101

Keywords: Infectious Diseases, Issue 83, transmissible spongiform encephalopathy, sheep, atypical scrapie, classical scrapie, neurologic examination, scratch test, menace response, blindfolding

Date Published: 1/19/2014

Citation: Konold, T., Phelan, L. Clinical Examination Protocol to Detect Atypical and Classical Scrapie in Sheep. J. Vis. Exp. (83), e51101, doi:10.3791/51101 (2014).

\section{Abstract}

The diagnosis of scrapie, a transmissible spongiform encephalopathy (TSEs) of sheep and goats, is currently based on the detection of diseaseassociated prion protein by post mortem tests. Unless a random sample of the sheep or goat population is actively monitored for scrapie, identification of scrapie cases relies on the reporting of clinical suspects, which is dependent on the individual's familiarization with the disease and ability to recognize clinical signs associated with scrapie. Scrapie may not be considered in the differential diagnosis of neurological diseases in small ruminants, particularly in countries with low scrapie prevalence, or not recognized if it presents as nonpruritic form like atypical scrapie. To aid in the identification of clinical suspects, a short examination protocol is presented to assess the display of specific clinical signs associated with pruritic and nonpruritic forms of TSEs in sheep, which could also be applied to goats. This includes assessment of behavior, vision (by testing of the menace response), pruritus (by testing the response to scratching), and movement (with and without blindfolding). This may lead to a more detailed neurologic examination of reporting animals as scrapie suspects. It could also be used in experimental TSE studies of sheep or goats to evaluate disease progression or to identify clinical end-point.

\section{Video Link}

The video component of this article can be found at https://www.jove.com/video/51101/

\section{Introduction}

Scrapie is a transmissible spongiform encephalopathy (TSE) of sheep and goats, which belongs to the group of protein misfolding disorders, like bovine spongiform encephalopathy (BSE) in cattle, chronic wasting disease in deer, and Creutzfeldt-Jakob disease in humans. The diagnosis is made post mortem by detecting the abnormal form of the prion protein, which is proteinase-resistant, usually in the brain. Two major naturally occurring scrapie forms can be distinguished, which are epidemiologically, pathologically, and biochemically different: classical scrapie and atypical scrapie. In addition, a neurological disease with classical scrapie-like clinical signs can be induced in sheep and goats by experimental infection with the BSE agent ${ }^{6,10-11}$. In fact, naturally occurring BSE has been diagnosed in goats ${ }^{3,17}$. Control of classical scrapie (and BSE in sheep if it occurred naturally) has been achieved by breeding for genetic resistance targeting the prion protein gene, but this is more difficult for atypical scrapie, which is believed to be a sporadic disease and has been diagnosed in countries considered to be free from classical scrapie, e.g. New Zealand ${ }^{7}$.

Scrapie is a neurologic disease of adult sheep and goats resulting in changes in behavior, sensation, and movement, but the disease is slowly progressive, and clinical signs are often not recognized because the animal keeper or veterinary surgeon may not be familiar with the clinical signs, or scrapie is not considered at all in the differential diagnosis of neurologic diseases in small ruminants.

The proposed examination protocol was designed to aid in the clinical diagnosis of scrapie with two purposes in mind: it should be considerably brief to allow scanning of many animals within a short period of time but specific enough to allow detection of scrapie-affected animals, which is achieved by assessing the presence of clinical signs frequently encountered in scrapie-affected animals. This protocol is a slight modification from the one used to monitor goats for signs of classical scrapie ${ }^{11}$ and includes blindfolding of the animal, which may exacerbate clinical signs not normally associated with scrapie ${ }^{9}$.

\section{Protocol}

\section{Assessment of Posture}

1. Assess the posture of the animal whilst undisturbed.

2. Animals affected by TSEs may stand with wide-based limbs, may appear hunched, or may stand with a low head carriage. 


\section{Assessment of Behavior}

1. Study the animal's behavior, when left alone, when approached, and when handled.

2. TSE-affected animals may display altered mentation by standing separately from other sheep or not immediately following other sheep.

3. When the animal is approached, it may run away and collapses, or it collapses to the floor when handled. Compulsive clockwise or counterclockwise circling may be seen in sheep with atypical scrapie.

\section{Menace Response Testing}

1. Hold the animal's head for testing of the menace response: move the hand or several fingers several times towards each eye of the animal to induce a blink response but be careful not to touch any facial hairs or create any air turbulence that may cause the animal to blink.

2. The menace response may be absent in scrapie-affected animals, particularly in sheep with atypical scrapie. Although testing of the menace response is the only cranial nerve testing used as part of the protocol, visual inspection of the face during this procedure may reveal other signs of neurologic deficits not normally seen in scrapie, e.g. facial paralysis. If the animal does not blink in response to the menace it is advisable to test the palpebral reflex by touching the eyelids to ensure that the animal's inability to blink is not caused by facial paralysis.

\section{Testing of the Scratch Response}

1. Ensure the animal is calm during this procedure because the test cannot be interpreted in a struggling animal.

2. Scratch areas of the back at the thoracic, lumbar, and sacral regions, pausing between each scratching episode.

3. If the animal responds with rhythmical head or body movements, or moves or licks its lips upon scratching of one particular area, repeat the scratch test to confirm reproducibility of the scratch response.

4. A repeatable response is usually elicited in animals that are pruritic, which usually includes sheep with classical scrapie or goats with the pruritic form of scrapie, but there is generally no response in sheep with atypical scrapie unless other pruritic skin conditions or ectoparasites are present.

\section{Checking for Wool Loss and Skin Lesions}

1. Inspect the skin.

2. Pruritus due to scrapie usually causes hair loss, which may be on the poll, back, rump, side of chest or abdomen due to excessive rubbing or scratching where the underlying skin appears normal.

3. Abrasions may appear later if the animal has the opportunity to rub on sharp objects. Bite wounds may appear, particularly on the limbs, due to excessive nibbling of the limbs.

\section{Scoring Body Condition}

1. Assess the animal's body condition by palpating the dorsal and transverse processes of the lumbar vertebrae.

2. Scrapie may cause a progressive loss of body condition where the transverse processes of the lumbar vertebrae can be easily felt individually, and the ends of the processes appear sharp.

\section{Blindfolding the Animal}

1. Cover the animal's eyes with a hood, which can be made by using any nonsee-through bag where one corner is cut off to allow breathing of the animal.

2. Release the animal only if there are no safety concerns, and there is no risk of injury due to a panicking animal; otherwise keep hold of the sheep and study animal movements whilst still in control of the animal.

3. Blindfolding may exacerbate signs of a vestibular system or cerebellar dysfunction, which may be obscured by the animal's ability to compensate through its vision. Loss of balance, incoordination, or circling may be elicited, particularly in otherwise clinically unremarkable sheep with atypical scrapie.

\section{Assessment of the Animal's Movement}

1. Study the animal's gait and muscle movements when it moves freely.

2. Incoordination may be characterized by wide-based movements of the hind limbs, hopping with both hind limbs, or swaying excessively in the hind quarters, which may result in stumbling or briefly sitting down.

3. The presence of a head tremor is often subtle when the animal is undisturbed but may become more noticeable during or after completion of the clinical examination.

\section{Interpretation}

1. Consider the significance of the findings with respect to scrapie.

2. Although the presence of individual signs, such as a repeatable response to scratching, tremor, abnormal behavior, circling, collapsing episodes, ataxia, or an absent menace response, may have various causes, the combined occurrence of two or more of these signs is highly suggestive of scrapie in sheep or goats. 


\section{Representative Results}

Clinically healthy sheep. Poll Dorset wether from a scrapie-free flock intracerebrally inoculated with bovine brain of New Zealand origin and examined at 49 months post inoculation. This sheep has a normal posture, behaves normally, displays a normal menace response (Figure 1), does not react to the scratch test, has an intact fleece, and a very good body condition. It is clinically unremarkable when blindfolded and shows no signs of a movement disorder.

Atypical scrapie sheep presenting with the nonpruritic form of TSEs in sheep. Cheviot wether from a scrapie-free flock intracerebrally inoculated with brain homogenate from an atypical scrapie field case of the same prion protein genotype (AHQ/AHQ) and examined at 36 months post inoculation. This sheep also has a normal posture but behaves differently to the other sheep in the pen and circles in clockwise direction. It does not blink when the menace response is tested. Scratching does not elicit any response, and no fleece changes are apparent, but the body condition is only fair. It circles clockwise when blindfolded (Figure 2) and loses balance. The gait is ataxic, and a fine head tremor is visible.

Atypical BSE sheep presenting with the pruritic form of TSEs in sheep, which is clinically similar to the clinical presentation of classical scrapieaffected sheep. For this reason, a classical scrapie case is not included. Poll Dorset wether from a scrapie-free flock intracerebrally inoculated with bovine atypical (L-type) BSE brain homogenate and examined at 50 months post inoculation. This sheep stands slightly hunched, tends to keep the hind limbs wide-based and falls on the floor when caught. The menace response is weak. Scratching of various areas of the back elicits head or lip movements (Figure 3), and there is wool loss with minor skin trauma on the poll. The body condition is good. When blindfolded, it is reluctant to move and rests its rear end on the hurdle to maintain balance. The sheep is ataxic and displays fore limb hypermetria.

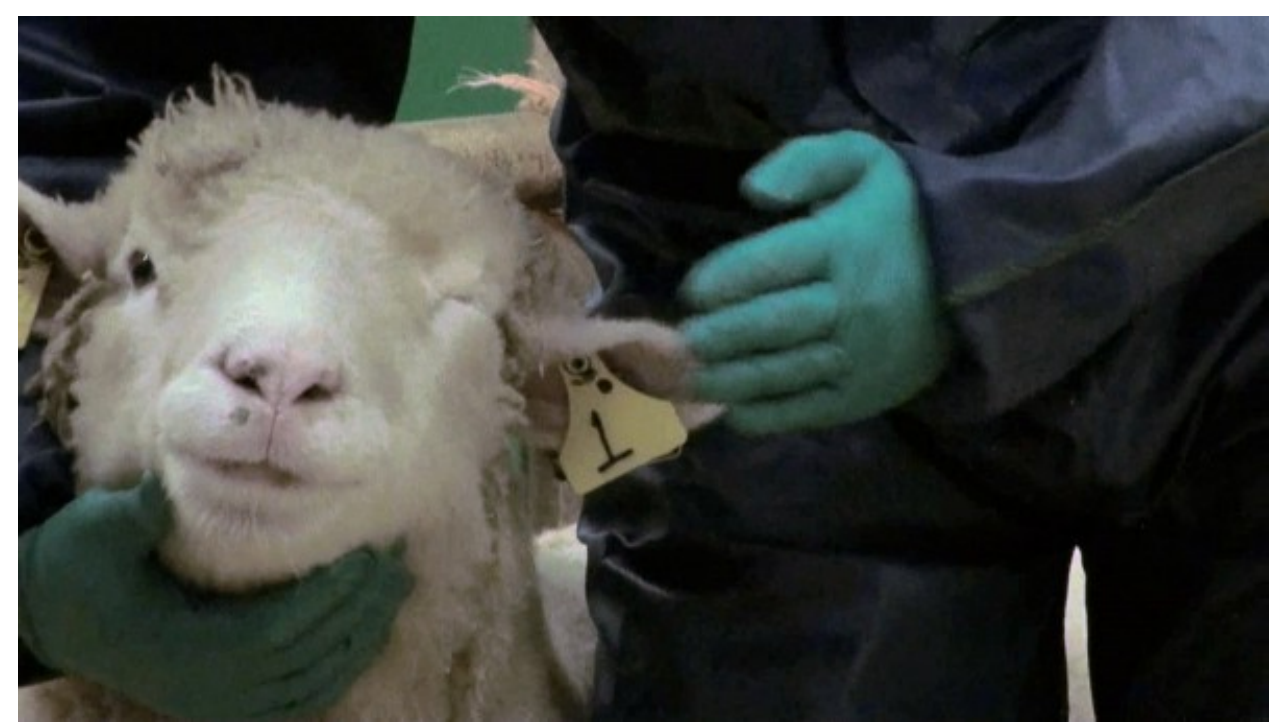

Figure 1. Menace response testing. Menacing gesture towards the animal's eye induces a blink response. Click here to view larger image.

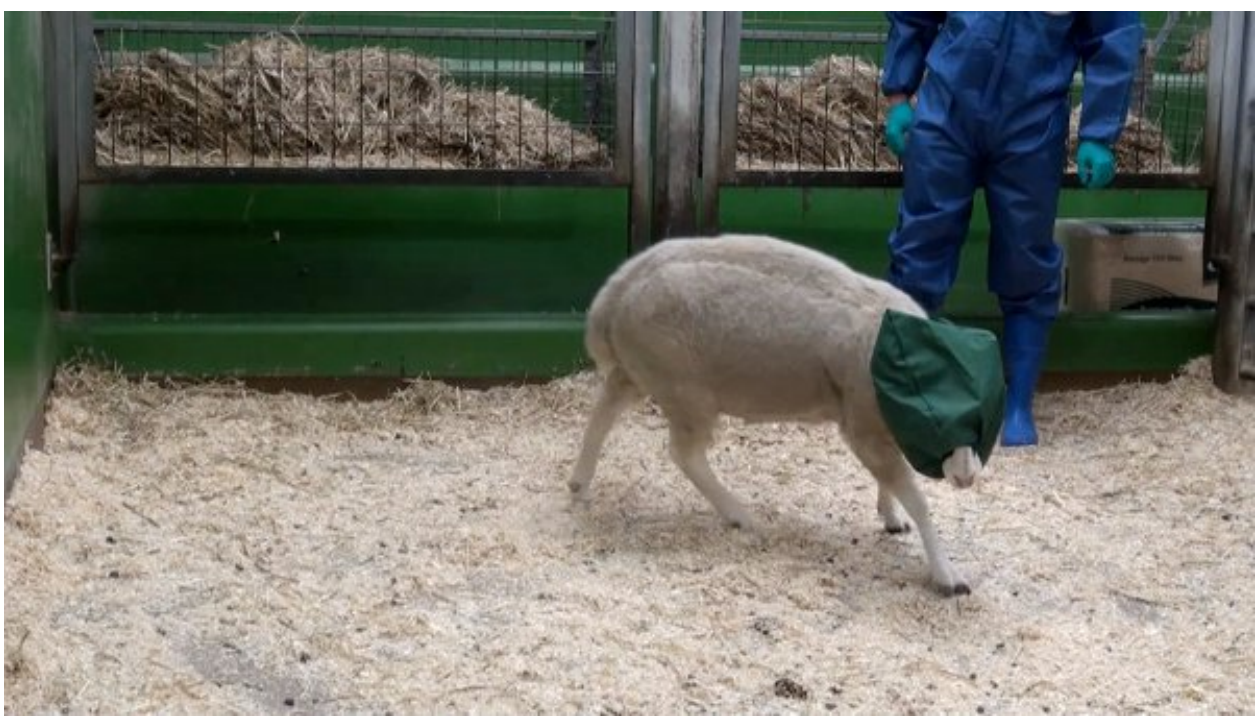

Figure 2. Blindfolding. Clockwise circling elicited by blindfolding the sheep with a hood. Click here to view larger image. 


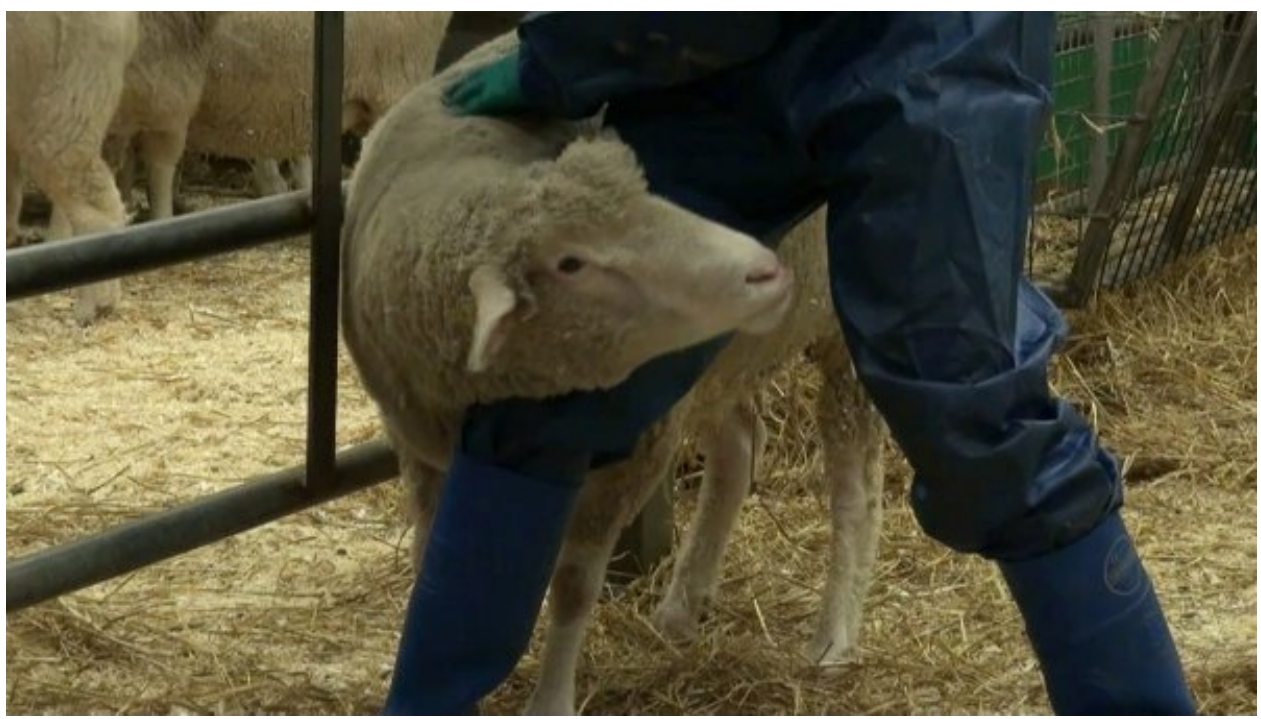

Figure 3. Testing of the scratch response. Scratching of the back elicits lip licking with sideward movements of the head Click here to view larger image.

\section{Discussion}

Scrapie is a listed disease by the World Health Organization for Animal Health (OIE), which, in the absence of a quick and reliable ante mortem test, relies on the farmers' or veterinarians' ability to recognize the clinical signs associated with this disease. Most scrapie cases, particularly atypical scrapie cases, however, are currently found by targeted TSE surveillance, i.e. by brain examination of small ruminants subject to slaughter or fallen stock animals. Breeding sheep for resistance to classical scrapie, which reduced the number of classical scrapie cases, most likely also reduced people's awareness for the disease so that it is less often considered in the differential diagnosis of neurologic disorders in sheep. Atypical scrapie also has a low prevalence with approximately five cases in abattoirs and eight cases in fallen stock per 10,000 tested in Europe $^{4}$. In addition, pruritus, usually evident as alopecia with or without excoriation, is often considered a characteristic sign of scrapie ${ }^{13}$. Thus, a neurologic disease without concomitant pruritus as observed in atypical scrapie ${ }^{12}$ or the nonpruritic form of caprine scrapie ${ }^{11}$ may not raise suspicion of scrapie.

This examination protocol was designed to identify clinical signs of TSE in sheep by evaluating specific clinical signs associated with various types of scrapie (atypical and classical scrapie, the latter represented by an atypical BSE-inoculated sheep, which presented with classical scrapie-like signs). In classical scrapie, the percentage of cases that displayed a positive scratch response ("nibble reflex") ranged from 50-77 in over 100 sheep studied ${ }^{5,8}$. Movement disorders can be observed in up to $81 \%$ of classical scrapie cases based on various studies ${ }^{18}$. Incoordination is also a major sign in various naturally occurring cases of atypical scrapie, often accompanied by behavioral changes ${ }^{1}$, but additional signs reported in both naturally and experimentally infected atypical scrapie sheep were circling and visual impairment ${ }^{12,15,16}$, which is why blindfolding and the testing of the menace response is included in the examination protocol. Scoring the body condition, for example using a 6 point scoring system ${ }^{14}$, enables evaluation of emaciation, which may be observed in scrapie-affected sheep ${ }^{5}$. Although clinical criteria to aid in the detection of scrapie in sheep have been proposed previously ${ }^{2}$, these only related to the detection of classical scrapie and took the clinical history into account, which may not be available if animals are presented at an abattoir.

The current protocol allows screening of many animals for signs of scrapie within a short period of time. Furthermore, the examination protocol can be applied to experimental studies of scrapie in sheep and goats to monitor clinical onset, progression, and end-point. However, it does not replace a more detailed physical and neurologic examination with appropriate recording of the clinical case history, possibly followed by ancillary diagnostic tests (examination of blood and cerebrospinal fluid; more sophisticated imaging techniques if appropriate), which may be necessary subsequently to rule out various other conditions in small ruminants that present with similar signs, such as metabolic or inflammatory neurological diseases, brain abscesses, or tumors.

Although a clinical examination is generally less sensitive in detecting TSE-affected animals than post mortem tests based on detection of prion protein $^{2,11}$, particularly in an early stage of the incubation period, this is currently the only detection method in live animals and most relevant to those countries that do not have a targeted surveillance system in place to identify TSE-affected small ruminants.

\section{Disclosures}

All procedures involving animals were carried out in accordance with the Animal (Scientific Procedures) Act 1986, under license from the UK Government Home Office.

The authors declare that they have no competing financial interests. 


\section{Acknowledgements}

The studies providing the animals were funded by the UK Department for Food, Environment and Rural Affairs and the European Union (through funding of the EU TSE Reference Laboratory). We acknowledge the support of the project managers, Dr. M. Simmons and Dr. M. Jeffrey.

\section{References}

1. Benestad, S. L., Arsac, J. N., Goldmann, W., \& Nöremark, M. Atypical/Nor98 scrapie: properties of the agent, genetics, and epidemiology. Vet. Res. 39 (4), 19 (2008).

2. D'Angelo, A., Maurella, C., et al. Assessment of clinical criteria to diagnose scrapie in Italy. Vet. J. 174 (1), 106-112 (2007).

3. Eloit, M., Adjou, K., et al. BSE agent signatures in a goat. Vet. Rec. 156 (16), 523-524 (2005).

4. Fediaevsky, A., Maurella, C., et al. The prevalence of atypical scrapie in sheep from positive flocks is not higher than in the general sheep population in 11 European countries. BMC Vet. Res. 6, 9 (2010).

5. Healy, A. M., Weavers, E., et al. The clinical neurology of scrapie in Irish sheep. J. Vet. Intern. Med. 17 (6), $908-916$ (2003).

6. Houston, E. F. \& Gravenor, M. B. Clinical signs in sheep experimentally infected with scrapie and BSE. Vet. Rec. 152 (11), $333-334$ (2003).

7. Kittelberger, R., Chaplin, M. J., et al. Atypical scrapie/Nor98 in a sheep from New Zealand. J. Vet. Diagn. Invest. 22 (6), $863-875$ (2010).

8. Konold, T., Bone, G., et al. Associations of clinical signs and prion protein genotypes in British sheep with scrapie. Dtsch. Tierärztl. Wochenschr. 116 (11), 380-388 (2009).

9. Konold, T., Bone, G., et al. Scrapie in goats. Vet. Rec. 161 (11), 395-396 (2007).

10. Konold, T., Bone, G., et al. Pruritus is a common feature in sheep infected with the BSE agent. BMC Vet. Res. 4, 16 (2008).

11. Konold, T., Bone, G. E., et al. Monitoring of clinical signs in goats with transmissible spongiform encephalopathies. BMC Vet. Res. 6, 13 (2010).

12. Konold, T., Davis, A., et al. Clinical findings in two cases of atypical scrapie in sheep: a case report. BMC Vet. Res. 3, 2 (2007).

13. Passler, T., Walz, P. H., \& Pugh, D. G. Diseases of the Neurologic System. In: Sheep and Goat Medicine. 2nd edn. Pugh, D. G. \& Baird, A. N., eds., W.B. Saunders, Saint Louis, 361-405 (2012).

14. Russel, A. Body condition scoring of sheep. In Pract. 6 (3), 91-93 (1984).

15. Simmons, H. A., Simmons, M. M., et al. Atypical scrapie in sheep from a UK research flock which is free from classical scrapie. BMC Vet. Res. 5, 8 (2009).

16. Simmons, M. M., Konold, T., et al. The natural atypical scrapie phenotype is preserved on experimental transmission and sub-passage in PRNP homologous sheep. BMC Vet. Res. 6, 14 (2010).

17. Spiropoulos, J., Lockey, R., et al. Isolation of prion with BSE properties from farmed goat. Emerg. Infect. Dis. 17 (12), $2253-2261$ (2011).

18. Ulvund, M. J. Clinical Findings in Scrapie. In: Prions in humans and animals. Hörnlimann, B., Riesner, D., \& Kretzschmar, H., eds., de Gruyter, Berlin, 398-407 (2006). 